\title{
Das letzte deutsch-deutsche Rentenkapitel: der Härtefallfonds
}

\author{
In der aktuellen Wahlperiode wird diskutiert, einen Fonds für Härtefälle aus der \\ Rentenüberleitung einzurichten. Damit soll die Deutsche Einheit in der Rentenfrage vollendet \\ werden. Dabei stellt sich die Frage, ob Ungerechtigkeiten aus der Rentenüberleitung \\ entstanden sind. Entgegen der in der Öffentlichkeit vorgebrachten These, dass erhebliche \\ Ungerechtigkeiten vorliegen, kann diese Analyse zeigen, dass zum Beispiel bei den in der \\ DDR geschiedenen Frauen keine ökonomische und politische Diskriminierung vorliegt. Die \\ Einrichtung eines Härtefallfonds erscheint dementsprechend als eine Scheinlösung, um eine \\ Befriedung betroffener Gruppen sicherzustellen.
}

\begin{abstract}
Nachdem in der 18. Wahlperiode im Deutschen Bundestag das Rentenüberleitungs-Abschlussgesetz verabschiedet wurde - und damit die Rentenangleichung spätestens im Jahr 2024 vollendet sein wird -, befassen sich in der aktuellen 19. Wahlperiode, in die auch die dreißigsten Jubiläen der friedlichen Revolution und der deutschen Einheit fallen, die Bundesregierung und der Deutsche Bundestag auch mit Fragen und besonderen Problemen, die sich aus der Rentenüberleitung ergeben. Mit der Einführung eines Härtefallfonds soll für Berufsund Personengruppen, die im Einigungsvertrag bei der Rentenüberleitung nicht im Sinne der Betroffenen berücksichtigt wurden, ein finanzieller Ausgleich außerhalb des Rentenrechts geschaffen werden. Im Koalitionsvertrag von CDU, CSU und SPD finden sich dazu zwei kurze Sätze: „Für Härtefälle in der Grundsicherung im Rentenüberleitungsprozess wollen wir einen Ausgleich durch eine Fondslösung schaffen. Entsprechendes wol-
\end{abstract}

(C) Der/die Autor(en) 2020. Open Access: Dieser Artikel wird unter der Creative Commons Namensnennung 4.0 International Lizenz (https:// creativecommons.org/licenses/by/4.0/deed.de) veröffentlicht.

Open Access wird durch die ZBW - Leibniz-Informationszentrum Wirtschaft gefördert.

* Der Autor vertritt hier seine persönliche Auffassung.

Dr. Alexander Mislin ist Regierungsdirektor im Bundesministerium der Finanzen und zurzeit Referent in der CDU/CSU-Fraktion im Deutschen Bundestag. len wir auch für die Gruppe der Spätaussiedler und der jüdischen Kontingentflüchtlinge prüfen." (Koalitionsvertrag, 2018, 93). Zur Umsetzung des Vorhabens wurde im Dezember 2018 eine Bund-Länder-Arbeitsgruppe eingesetzt. Mit der Feststellung, einen „Fonds für Härten in der Rentenüberleitung“ einrichten zu wollen, liegt ein vorläufiges Ergebnis vor. Für die Finanzierung ist aber noch offen, ob der Bund allein bezahlt oder ob die ostdeutschen Bundesländer sich beteiligen.

Politisch betrachtet muss für die Einrichtung eines Härtefallfonds ein Kriterium maßgeblich sein, damit er erfolgreich ist. Er muss eine Befriedung mit den betroffenen Gruppen sicherstellen. Folglich darf die Härtefallregelung nicht dazu führen, dass neue Ungerechtigkeiten entstehen. Hinzu kommt, dass mit der Einführung der Grundrente ab dem 1. Januar 2021 bereits eine Mindestabsicherung für Versicherte mit einer Beitragsdauer von 33 Versicherungsjahren eingeführt wird (Mislin, 2019). Daher dürfte sich der Kreis der Berechtigten für einen Härtefallfonds im Sinne des Koalitionsvertrags verkleinern. Mit dem Härtefallfonds sollen Personen, die sich - dann nach Einführung einer Grundrente - in der Grundsicherung befinden, eine zusätzliche Transferzahlung erhalten, wenn sie denn zu einer, von der Politik bestimmten, Berufs- und Personengruppe zählen, die durch die Rentenüberleitung eine besondere „Härte“ erfahren hat. Ein Blick auf die Statistik der Grundsicherung im Alter und bei Erwerbsminderung öffnet die Augen für die Größe des Problems (vgl. Abbildung 1).

Schaut man sich die Quoten der Empfänger*innen von Grundsicherung im Alter und bei Erwerbsminderung für die alten und neuen Länder für den Zeitraum 2003 bis 2018 an, so stellt man fest, dass Altersarmut für die über- 
Abbildung 1

Quote der Empfänger*innen der Grundsicherung im Alter und bei Erwerbsminderung ${ }^{1}$

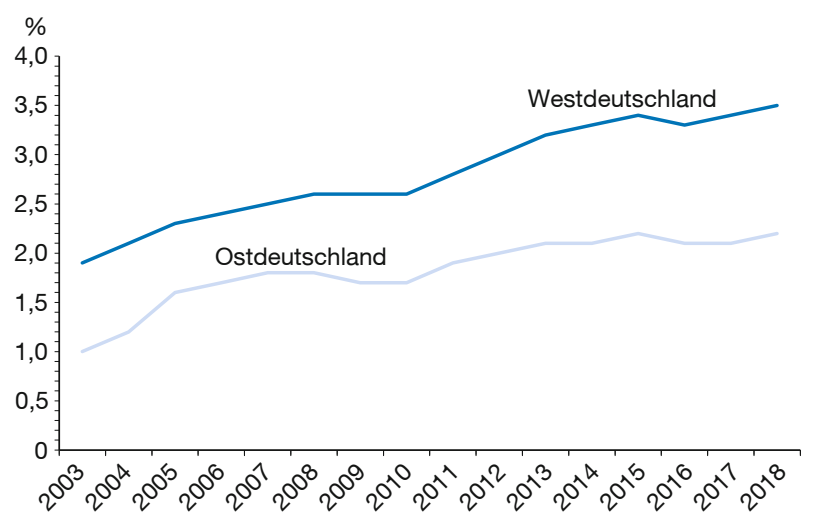

${ }^{1}$ Die Quote bezieht sich auf die Bevölkerung im Alter oberhalb der Regelaltersgrenze (für das Jahr 2018 = 65 Jahre und 7 Monate).

Quelle: Statistisches Bundesamt (Destatis), 2020 - Statistik der Grundsicherung im Alter und bei Erwerbsminderung.

wiegende Mehrheit der Bestandsrentner*innen in den neuen Ländern kein Problem ist. ${ }^{1}$

\section{Die Rentenüberleitung}

Bereits bei den Verhandlungen zum Einigungsvertrag haben sich die Vertragsparteien darauf verständigt, dass die Alterssicherung für alle in der DDR zurückgelegten Zeiten einheitlich in der gesetzlichen Rentenversicherung vorzunehmen ist. Diese sogenannte Systementscheidung wurde auch durch die Rechtsprechung des Ersten Senats des Bundesverfassungsgerichts am 28. April 1999 bestätigt. Am 21. Juni 1991 hat der Deutsche Bundestag mit dem Koalitionsantrag der Fraktionen der CDU/CSU und der FDP das Renten-Überleitungsgesetz (RÜG) beschlossen. Das RÜG wurde am 25. Juli 1991 ausgefertigt und trat am 1. Januar 1992 in Kraft. Damit konnte Artikel 30 Abs. 5 des Einigungsvertrags umgesetzt werden, wonach die gesetzliche Rentenversicherung nach Sozialgesetzbuch Sechs (SGB VI) auf das Beitrittsgebiet überzuleiten ist. Seit dem 1. Januar 1992 gilt das SGB VI für ganz Deutschland. Für Versicherte, deren Rentenbeginn in den Zeitraum 1. Januar 1992 bis 30. Juni 1995 fiel, wurde die Rentenzahlung nach den Grundsätzen des Rentenrechts der ehemaligen DDR gezahlt, wenn sich für die Versicherten daraus eine höhere Rente als nach dem SGB VI ergab. Mit der Überführung der Sozialpflichtversicherung und der Freiwilligen Zusatzrentenversicherung (FZR) wurden auch die Zusatz- und Sonderversorgungssysteme der ehemaligen DDR in die Deutsche Rentenversicherung

1 Neue Bundesländer einschließlich Berlin.
Abbildung 2

Bundesmittel an die gesetzliche Rentenversicherung: Erstattungen im Rahmen des AAÜG

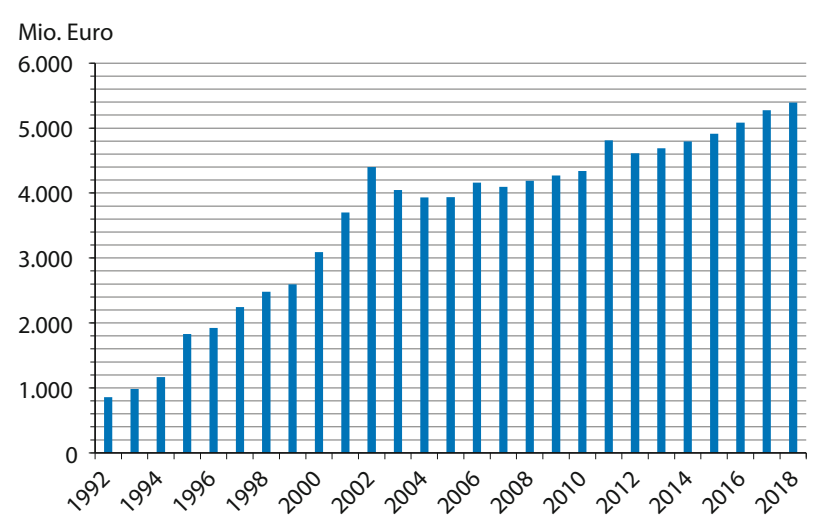

AAÜG = Anspruchs- und Anwartschaftsüberführungsgesetz.

Quelle: Rentenversicherung in Zeitreihen, Oktober 2019, 239. Deutsche Rentenversicherung Bund.

überführt. Das Anspruchs- und Anwartschaftsüberführungsgesetz (AAÜG) gibt dafür den formalen Rahmen. Anlage 1 des AAÜG führt 27 Zusatzversorgungssysteme und Anlage 2 des AAÜG vier Sonderversorgungssysteme auf. Bund und Länder teilen sich die Kosten, indem sie den Betrag, der nicht auf die Sozialpflichtversicherung der DDR entfällt, an die Deutsche Rentenversicherung erstatten. Dabei übernimmt der Bund bei der Zusatzversorgung zurzeit $40 \%$ der Kosten, während die ostdeutschen Länder $60 \%$ tragen und dem Bund erstatten. Von 1992 bis 2018 sind rund 100 Mrd. Euro für AAÜG-Leistungen an die gesetzliche Rentenversicherung erstattet worden (vgl. Abbildung 2). Ab dem 1. Januar 2021 wird sich der Bund abermals stärker an den Kosten aus den Zusatzversorgungssystemen der ehemaligen DDR beteiligen. ${ }^{2}$ Der Anteil soll dann auf $50 \%$ aufgestockt werden (Deutscher Bundestag, 2020).

Im Gegenzug zum statischen Rentensystem der ehemaligen DDR ist die dynamische Rentenberechnung mit zwei unterschiedlichen Rentenwerten und einem Umrechnungsfaktor, der den Unterschied zwischen dem Durchschnittsgehalt Ost mit dem Durchschnittsgehalt West ausgleichen soll, aus ostdeutscher Sicht vorteilhaft (Genett, 2005; Mislin, 2017). Die Eingliederung der ostdeutschen Rentner*innen in die Deutsche Rentenversicherung ist eine der großen Leistungen der deutschen Einheit. Die Rentenüberleitung ist insgesamt betrachtet sehr gut gelungen. Seit dem 1. Juli 2020 liegt der aktuelle Rentenwert

2 Der Bund hatte bereits seinen Anteil von ursprünglich $33 \%$ auf $36 \%$ im Jahr 2008 und seit dem Jahr 2010 auf $40 \%$ aufgestockt. 
bei 34,19 Euro und der Rentenwert (Ost) bei 33,23 Euro. Damit erreicht im Jahr 2020 der Rentenwert (Ost) 97,2\% des aktuellen Rentenwerts. Mit dem Beschluss vom 1. Juni 2017 hat der Deutsche Bundestag die Rentenangleichung auf den Weg gebracht (Mislin, 2017).

\section{Probleme aus der Rentenüberleitung?}

Mehrere Berufs- und Personengruppen der ehemaligen DDR sehen die Überleitung der DDR-Renten in die bundesdeutsche gesetzliche Rentenversicherung als nicht gelungen an. So wurden seit der Rentenüberleitung im Jahr 1992 wiederholt zahlreiche Einzelforderungen zu Änderungen im Recht der Rentenüberleitung von Betroffenen selbst oder im parlamentarischen Raum vorgetragen (Deutscher Bundestag, 2018b). Prominent in der öffentlichen Debatte sind hier unter anderem neben den ehemaligen Mitarbeiter*innen der Deutschen Reichsbahn, den Bergleuten, Postmitarbeiter*innen, Balletttänzer*innen, Pflegekräften auch die in der DDR geschiedenen Frauen zu zählen. Die Erfüllung der Forderungen bzw. die Beseitigung der vermeintlichen Defizite der Rentenüberleitung ist aus Sicht der betroffenen Gruppen das Kriterium für die Vollendung der inneren Einheit und damit für eine abschlieBende Befriedung der deutsch-deutschen Rentenfrage.

Juristisch sind aber sämtliche Fälle ausgeurteilt. Es gibt von Rechtswegen keine Veranlassung, dass die Politik eine Ausgleichslösung herbeiführt. Das Bundesverfassungsgericht weist in seinem Urteil aus dem Jahre 1999 darauf hin, dass es für die Gesetzgebung keine zwingende verfassungsrechtliche Verpflichtung gibt, die DDR-Zusatzrenten so zu betrachten, als ob die ehemaligen DDR-Bürger*innen inr Leben in der Bundesrepublik Deutschland verbracht hätten, und daraus einen Rechtsanspruch abzuleiten: „Bei den mit einem solchen Systemwechsel verbundenen Übergangsproblemen für diejenigen Personen, die bereits Ansprüche oder Anwartschaften erworben haben, muß freilich die Eigentumsgarantie des Art. 14 Abs. 1 Satz 1 GG berücksichtigt werden. Diese verleiht der individuellen Rechtsposition aber keinen absoluten Schutz. Das gilt insbesondere, wenn der Gesetzgeber vor der Aufgabe steht, ein System, das in eine von ganz anderen Prinzipien geleitete Rechtsordnung integriert war, an die Rechtsordnung der Bundesrepublik Deutschland anzupassen. Er ist dabei verfassungsrechtlich nicht verpflichtet, die Berechtigten aus Versorgungssystemen der Deutschen Demokratischen Republik so zu behandeln, als hätten sie ihre Erwerbsbiografie in der Bundesrepublik Deutschland zurückgelegt." (Bundesverfassungsgericht, 1999, 31).

Die Bundesregierung hat in der Vergangenheit keinen Handlungsbedarf gesehen, zumal selektive Regelungen für einzelne Berufs- und Personengruppen keinen Rechts- frieden schaffen, sondern nur die Unzufriedenheit bei den Nichtbegünstigten vergrößern. Problematisch ist zudem, dass es keine verlässliche Datenbasis über die Größe der betroffenen Gruppen gibt. Das Statistische Bundesamt und auch die Deutsche Rentenversicherung Bund können keine validen Zahlen angeben, wie groß der Kreis der Betroffenen Berufs- und Personengruppen ist. Angaben der Betroffenen („Runder Tisch der Berufs- und Personengruppen der ehemaligen DDR“) zufolge sollen rund 500.000 Personen Ansprüche auf Ausgleichsleistungen fordern. Exemplarisch soll an dieser Stelle die mit etwa 200.000 Betroffenen zahlenmäßig größte Gruppe betrachtet werden, und zwar die in der DDR geschiedenen Frauen, die in der politischen Debatte als Härtefallgruppe identifiziert wird und daher als potenziell Begünstigte für eine Fondslösung in Betracht kommt (Bundesrat, 2004; Deutscher Bundestag, 2017, 2018a). ${ }^{3}$

\section{Die Gruppe der in der DDR geschiedenen Frauen}

Bei der Gruppe handelt es sich um Frauen, die vor dem 1. Januar 1992 nach DDR-Recht geschieden wurden. ${ }^{4}$ Das Scheidungsrecht der ehemaligen DDR war im Familiengesetzbuch (FGB) geregelt. Ehegattenunterhaltsansprüche waren auf zwei Jahre befristet. Die Geschiedenenwitwenrente sowie einen Versorgungsausgleich, der im Scheidungsfall die Rentenansprüche aufteilt, gab es nicht. Die gesellschaftliche Realität in der ehemaligen DDR war dadurch geprägt, dass der Staat mit einem umfassenden System von Betreuungseinrichtungen es den Müttern ermöglichte Erwerbs- und Familienarbeit miteinander zu verbinden (Eichenhofer, 1995).

Für Ehen, die in den alten Ländern bis zum 30.6.1977 geschieden wurden, konnte die Geschiedenenwitwenrente beantragt werden, und zwar dann, wenn zuvor Unterhalt geleistet wurde. Seit dem 1. Juli 1977 gilt in der Bundesrepublik Deutschland das „Erste Gesetz zur Reform des Ehe- und Familienrechts“, das den sogenannten Versorgungsausgleich im Scheidungsfall durch eine Anrechnung von Ehejahren auf die spätere Rente bestimmt. Nach § 243a SGB VI wurde durch die Gesetzgebung festgelegt, dass geschiedene Frauen in den neuen Ländern keine Geschiedenenwitwenrente erhalten. Überdies ist im Einigungsvertrag geregelt, dass es keinen Versorgungsausgleich für in der DDR geschiedene Frauen gibt. Mit dem Gesetz zur Überleitung des Versorgungsausgleichs auf das Beitrittsgebiet (Versorgungsausgleichs-Überleitungsgesetz - VAÜG) gilt seit dem 1. Januar 1992 auch

3 Auch DDR-geschiedene Männer könnten als Härtefallgruppe in Betracht kommen. In diesem Beitrag soll auf die politisch erheblich wirkmächtigere Gruppe DDR-geschiedene Frauen eingegangen werden.

4 Nach Art. 234 §§ 5 und 6 EGBGB gilt das Scheidungs- bzw. Unterhaltsrecht der ehemaligen DDR nach dem FGB fort. 
in Ostdeutschland eine ebensolche Regelung. Eine nachträgliche oder gar rückwirkende Regelung hat die Gesetzgebung ausgeschlossen (Art. 234 § 6 EGBGB).

Die Betroffenen streben eine Anerkennung ihrer Familienarbeit an, indem sie eine rückwirkende Nachholung des Versorgungsausgleichs zu Lasten ihrer geschiedenen Ehemänner, hilfweise eines fiktiven Versorgungsausgleichs auf Kosten der Rentenkassen bzw. eine entsprechende staatliche „Entschädigungsleistung” fordern. Der Verein der in der DDR geschiedenen Frauen, der die Interessen der Betroffenen gegenüber der Politik vertritt, setzt sich dafür ein, dass die Lebensleistung, vor allem in Form der Familienarbeit, rentenrechtlich anerkannt wird. Die Betroffenen sehen erhebliche frauenspezifische Nachteile. So wurde häufig die Berufstätigkeit aufgegeben oder eingeschränkt für die Kinderbetreuung und anderweitige familiäre Aufgaben. Dem ist allerdings entgegenzuhalten, dass die Anerkennung der Familien- bzw. Erziehungsarbeit bereits mit der Gutschrift der Kindererziehungszeiten auf die gesetzliche Rente erfolgt. Mit den Gesetzen über Leistungsverbesserungen in der gesetzlichen Rentenversicherung zum 1. Juli 2014 (Mütterrente I) und zum 1. Januar 2019 (Mütterrente II) erfolgten bereits Verbesserungen, indem nun Kindererziehungszeiten für die Erziehung von vor 1992 geborenen Kindern von einem auf maximal zweieinhalb Jahre verlängert wurden.

Auch die Frauen, die im Betrieb beim Ehemann mitgeholfen haben (sogenannte mithelfende Ehefrauen) und freiwillige Beiträge zur Sozialversicherung eingezahlt haben, erfahren in der Regel keine Schlechterstellung gegenüber dem ehemaligen DDR-Rentenrecht. Bezogen auf das SGB VI können freiwillig gezahlte Beiträge unterhalb des Mindestbeitrags nicht zu dynamischen Rentenleistungen führen (§ 248 Abs. 3 Satz 2 Nr. 3 SGB VI i.V.m. Anlage 11 SGB VI), gleichwohl aber zu statischen Zusatzleistungen, die zur Rente gezahlt werden. ${ }^{5}$ Sie richten sich nach dem Alter der Versicherten zum Zeitpunkt der Beitragszahlung und liegen zwischen 1,6667\% und 0,8333\% des Beitrags ( $\$ 269$ Abs. 1 SGB VI). Nach DDRRecht wurde ein Steigerungsbetrag von $0,85 \%$ für die Rente berücksichtigt.

Die ökonomisch interessante Frage, die sich bei der Einführung eines Härtefallfonds stellt, ist, ob das Merkmal „Scheidung" eine ungleiche Behandlung von Frauen mit einer ähnlichen Erwerbsbiografie rechtfertigt. Der Versuch, die Gruppe der in der DDR geschiedenen Frauen finanziell besserzustellen, in dem der Versorgungsausgleich rückwirkend eingeführt wird, ist aus verfassungs-

5 In der DDR galt ab dem 1.1.1962 ein Mindestpflichtbeitrag in Höhe von 15 Mark der DDR (20\% von 75 Mark der DDR).
Abbildung 3

Quote der Empfängerinnen der Grundsicherung im Alter und bei Erwerbsminderung ${ }^{1}$

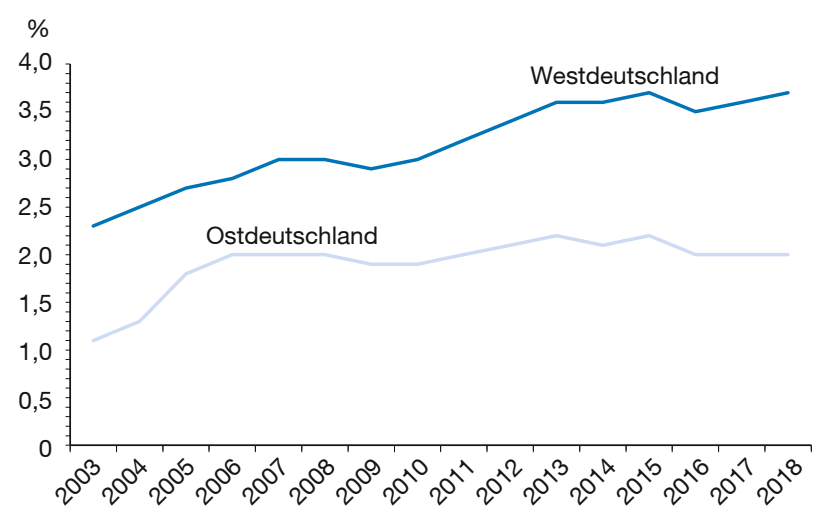

${ }^{1}$ Die Quote bezieht sich auf die weibliche Bevölkerung im Alter oberhalb der Regelaltersgrenze (für das Jahr 2018 = 65 Jahre und 7 Monate).

Quelle: Statistisches Bundesamt (Destatis), 2020 - Statistik der Grundsicherung im Alter und bei Erwerbsminderung.

rechtlichen Gründen nicht machbar (sogenanntes Rückwirkungsverbot). Die in der DDR geschiedenen Männer finanziell heranzuziehen ist nicht möglich, weil die damals in der DDR gültigen Gesetze sich nicht nachträglich zu deren Nachteil ändern lassen. Die geschiedenen Ehemänner könnten sich auf den Grundsatz des Vertrauensschutzes und der Rechtssicherheit berufen (Art. 20 Abs. $3 \mathrm{GG}$ ). Schließlich handelt es sich beim Versorgungsausgleich um einen innerehelichen Vermögensaustausch zu Lasten des Ehegatten mit den höheren Versorgungsbezügen. Zudem wäre es gegenüber denjenigen Frauen diskriminierend, die in Westdeutschland vor 1977 geschieden wurden - und deshalb ebenfalls keinen Anspruch auf den Versorgungsausgleich haben. Eine Lösung, die sich in die bestehende Rechtssystematik einfügt, hat sich bislang als schwierig erwiesen.

Für Heinke und Fuchsloch (2002) ist die Regelung des $\S 243 a$ SGB VI verfassungswidrig, und zwar dahingehend, dass ein Verstoß gegen Art. 3 Abs. 1 GG vorliegt, sofern eine nennenswerte Zahl von Frauen betroffen ist. Aus heutiger Sicht stellt sich die Sachlage folgendermaßen dar. Von 1977 bis 1991 wurden nach dem Recht der ehemaligen DDR 668.993 Ehen geschieden (Statistisches Bundesamt, 2019). Die Kausalverknüpfung, dass Frauen, die in diesem Zeitraum in der DDR geschieden wurden, wegen des fehlenden Versorgungsausgleichs heute in Altersarmut leben, ist wenig überzeugend, da die ostdeutschen Rentnerinnen in der Regel weit weniger von Altersarmut betroffen sind als westdeutsche Rentnerinnen (vgl. Abbildung 3). 
Mit Blick auf die unklare Finanzierung hat die Bundesregierung in der Vergangenheit eine Lösung bzw. eine Befriedung des Sachverhalts durch einen Ausgleichsfonds für Härtefälle abgelehnt (Deutscher Bundestag, 1996). Zudem ist mit dem Beschluss der 3. Kammer des Ersten Senats des Bundesverfassungsgerichts vom 2. Juni 2003 auf juristischem Wege die Problematik abgeschlossen.

Auf internationaler Ebene sieht der Ausschuss zum Übereinkommen der Vereinten Nationen zur Beseitigung jeder Form von Diskriminierung der Frauen (Committee on the Elimination of Discrimination against Women, CEDAW) Handlungsbedarf bei der Gruppe der in der DDR geschiedenen Frauen. In seinem Übereinkommen vom 9. März 2017 hat sich der CEDAW dafür ausgesprochen, dass Deutschland ein staatliches Entschädigungsmodell einrichten sollte, um Wiedergutmachung zu leisten, in dem die Renten von in der ehemaligen DDR geschiedenen Frauen ergänzt werden (Empfehlung Nr. 50d). Im Rahmen des Berichtsverfahrens lehnt die Bundesregierung in ihrem Zwischenbericht eine rentenrechtliche Sonderstellung für DDR-Geschiedene aufgrund von verfassungsrechtlichen Problemen ab (CEDAW, 2019).

\section{Fazit}

Die Analyse hat gezeigt, dass es bei der Einrichtung eines Härtefallfonds weder um das Problem der Altersarmut noch um die Beseitigung rechtlicher Lücken bei der Rentenüberleitung geht. Eine Ausgleichslösung ist im Grunde nicht notwendig. Vielmehr geht es hier um die ausschließlich monetäre Befriedung spezieller gesellschaftlicher Gruppen. Die meisten Rentner*innen der betroffenen Berufs- und Personengruppen würden allerdings von einem Härtefallfonds, wie inn der Koalitionsvertrag vorsieht, nicht profitieren. Wenn nun aber nur ein Bruchteil der Betroffenen in den Genuss einer Fondslösung kommen würde, würde die Aussicht auf eine gesamtgesellschaftliche Befriedung nicht mehr gegeben sein. Unter den gegebenen politischen und ökonomi- schen Überlegungen wäre die First-Best-Lösung, von dem Vorhaben im Koalitionsvertrag abzusehen.

\section{Literatur}

Bundesverfassungsgericht (1999), Urteil des Ersten Senats vom 28 April 1999 - 1 BvL 32/95 -, Rn. (1-201), http://www.bverfg.de/e/ Is19990428_1bvl003295.html (31. März 2020).

Bundesrat (2004), BR-Drucksache 677/04 vom 3. September 2004 - Antrag des Freistaates Sachsen, Entschließung des Bundesrates „Verbesserung der rentenrechtlichen Situation der im Beitrittsgebiet vor dem 01.01.1992 Geschiedenen“.

Deutscher Bundestag (1996), BT-Drucksache 13/6449 vom 27. Dezember 1996 - Antwort der Bundesregierung auf die Kleine Anfrage der Abgeordneten Christel Hanewinckel, Wolfgang Behrendt, ... - Rentenrechtliche Sicherung Altersgeschiedener der ehemaligen DDR.

Deutscher Bundestag (2017), BT-Drucksache 19/220 vom 11. Dezember 2017 - Antrag der Fraktion Die Linke: Forderung der Vereinten Nationen zu den in der DDR geschiedenen Frauen sofort umsetzen.

Deutscher Bundestag (2018a), BT-Drucksache 19/983 vom 28. Februar 2018 - Antrag der Fraktion Bündnis 90/Die Grünen: Eine Lösung für die rentenrechtliche Situation der in der DDR geschiedenen Frauen schaffen.

Deutscher Bundestag (2018b), Petitionsausschuss, Petition Id-Nr. 81823, https://epetitionen.bundestag.de/content/petitionen/_2018/_06/_26/ Petition_81823.html (13. Mai 2020).

Deutscher Bundestag (2020), BT-Drucksache 19/21753 vom 19. August 2020 - Gesetzentwurf der Bundesregierung, Entwurf eines Gesetzes zur finanziellen Entlastung der Kommunen und der neuen Länder.

CEDAW (2019), Interim Report by the Federal Republic of Germany, https://www.bmfsfj.de/blob/136168/41562bdf33d23798f1b1fcbb21f6 69fc/20190517-cedaw-zwischenbericht-englisch-data.pdf (31. Juli 2020).

Eichenhofer, E. (1995), Grundprobleme der Rentenüberleitung, Die Sozialgerichtsbarkeit, 42(11), 469-473.

Genett, T. (2005), Die Rentenfragen in den neuen Ländern 15 Jahre nach der Sozialunion, Deutschland Archiv, 38(3), 424-430.

Heinke, S. und C. Fuchsloch (2002), Die Nichteinführung der Geschiedenenwitwenrente für nach DDR-Recht geschiedene Ehegatten im Beitrittsgebiet - verfassungsgemäß?, Neue Justiz, 56(3), 113-117.

Koalitionsvertrag (2018), Ein neuer Aufbruch für Europa. Eine neue Dynamik für Deutschland. Ein neuer Zusammenhalt für unser Land, Koalitionsvertrag zwischen CDU, CSU und SPD, 19. Legislaturperiode, Berlin.

Mislin, A. (2017), Die Rentenberechnung in Deutschland - Rentenangleichung Ost-West, WiSt - Wirtschaftswissenschaftliches Studium, 46(11), 21-26.

Mislin, A. (2019), Die Grundrente - ein wirksames Instrument gegen Altersarmut in Ostdeutschland?, WiSt - Wirtschaftswissenschaftliches Studium, 48(6), 32-38.

Statistisches Bundesamt (2019), Eheschließungen, Ehescheidungen.

Title: The Last German-German Pension Chapter: A Fund for Social Hardships

Abstract: This paper analyses whether a fund for social hardships from the pension transition is politically an effective instrument for completing the German unity in the pension issue. For this purpose, one group ("women divorced under GDR law") is identified as a hardship case and analysed with regards to whether injustices from the pension transfer are present. Contrary to public opinion, the analysis shows that in the case of women divorced in the GDR, there is no economic or political discrimination. Overall, the transition of the German pension insurance to East Germany is a great success of the federal and state governments. In this case, a fund for social hardships is to be viewed as a false solution to the problem.

JEL Classification: D78, H55, H75 